\title{
ESTUDIO DE LA CINÉTICA DE DETERIORO DE PATACONES PREFRITOS CONGELADOS COMO ALIMENTO DERIVADO DEL PLÁTANO VERDE (MUSA PARADISIACA)
}

\section{Study Of The Kinetics Of Deterioration Of Frozen Pre-Fried Patacones As A Food Derived From Green Plantain (Musa Paradisiaca)}

\author{
${ }^{1}$ Cristina Muñoz Shugulí*, ${ }^{1}$ Cristian Patiño Vidal, ${ }^{2}$ Eduardo Muñoz Jácome
}

${ }^{1}$ Departamento de Ingeniería Química, Universidad de Santiago de Chile, Santiago, Chile.

${ }^{2}$ Facultad de Recursos Naturales, Escuela Superior Politécnica de Chimborazo, Riobamba, Ecuador.

cristina.munoz.as@gmail.com

\section{$R$ esumen}

El patacón es un producto elaborado a base de plátano verde y es uno de los alimentos más consumidos por países productores de plátano. Industrialmente, este podría constituir un alimento innovador de exportación, pero la producción y parámetros importantes de su vida útil no han sido estudiados. En este contexto, el objetivo del presente trabajo fue estudiar la cinética de algunos parámetros más importantes de conservación de patacones prefritos y congelados. Para esto, se realizó un estudio de vida útil acelerada a tres temperaturas de almacenamiento: $5{ }^{\circ} \mathrm{C}, 20^{\circ} \mathrm{C}$ y $30^{\circ} \mathrm{C}$. Se determinó el cambio en el brillo y color de los patacones a través un sistema de visión computacional y la pérdida de peso mediante método gravimétrico. El brillo $\left(\mathrm{L}^{*}\right)$ siguió una cinética de primer orden y fue ligeramente dependiente de la temperatura de almacenamiento mientras que el cambio de color $(\Delta \mathrm{E} 2000)$ y el porcentaje de pérdida de peso siguieron una cinética de orden cero, siendo el color el parámetro más susceptible a la temperatura de almacenamiento.

Palabras claves: cinética, patacones prefritos, color, pérdida de peso.

\section{A bstract}

Flat green banana chip "patacón" is a green banana based food and it is one of the most consumed products by banana producing countries. Industrially, this is an innovative exporting product for these countries but the production and important parameters of its shelf-life has not been studied. In this context, the aim of this work was study the kinetics of some of the most important parameters of conservation in pre-fried and frozen patacones. For this, an accelerated shelf-life study was carried out at three different storage temperatures: $5{ }^{\circ} \mathrm{C}, 20^{\circ} \mathrm{C}$ and $30^{\circ} \mathrm{C}$. Changes in lightness and color were measured by a computer vision system and a gravimetric method was used to calculate the weight loss percentage. Lightness $\left(\mathrm{L}^{*}\right)$ followed a first order kinetics order and was lightly dependent of storage temperature while color change $(\Delta \mathrm{E} 2000)$ and weight loss percentage followed a zero order kinetics, being color the most susceptible parameter to storage temperature.

Keywords: kinetics, prefried patacones, color, loss of weight. 


\section{INTRODUCCIÓN}

El plátano verde tiene gran importancia socioeconómica en varios países a escala mundial (1). Este constituye una fuente significativa de empleo e ingresos debido a su exportación y sobre todo al consumo masivo local ya que su aporte energético es fundamental en la dieta diaria de los habitantes de países productores de esta fruta $(2,3)$. Existe una gran variedad de plátanos, el más importante es el "Horn Plantain" conocido en Costa Rica como "Curarré" y en Ecuador como "Barraganete". También se encuentra otro tipo de plátano como "French Plantain" o "Plátano Dominico" y los clones conocidos como "Guineas"(4). La transformación del plátano verde, en cualquiera de sus variedades, se ha concentrado en actividades artesanales y pequeñas agroindustrias que utilizan diversas técnicas para obtener productos derivados tales como fritos (ej. chips con distintos sabores y condimentos, rebanadas con sal), fritos congelados (ej. tostones o patacones) y preparados (ej. harinas, pastas, tortillas) (5) que también son altamente consumidos y comercializados.

El patacón, conocido en otros países latinoamericanos como tostón, es elaborado a partir de trozos de plátano verde que son sometidos a fritura, prensado o formado y vuelven a ser fritos para obtener un producto final de color amarillo dorado con textura crujiente (4). Actualmente, los patacones se elaboran de forma casera para consumo inmediato y requieren mucho tiempo en su obtención, sin embargo la actual población demanda menos tiempo en la preparación de sus alimentos por lo que se propone productos pre-procesados. De forma adicional, alimentos con valor agregado como patacones prefritos y congelados podrían constituir un producto innovador de exportación. Sin embargo, la cadena de frío representa un aspecto muy importante para la calidad de estos (5); por tal razón la evaluación y seguimiento de ciertos parámetros de calidad a diferentes temperaturas de almacenamiento es sustancial en este producto.

Parámetros como el brillo, color y pérdida de peso en un alimento son indicadores de su deterioro. Además, estos son muy importantes en la aceptación del producto por parte de los consumidores, y constituyen una preocupación para la industria ya que se pueden ver comprometidos de forma legal y comercial (6). Adicionalmente, el proceso de congelación en productos con alto contenido de grasa como los pre fritos permite mantener e incluso prolongar su vida útil. Por esto, el objetivo del presente trabajo fue evaluar la cinética de deterioro con respecto al brillo, color y pérdida de peso en patacones prefritos y congelados durante su almacenamiento a tres distintas temperaturas mediante un estudio de vida útil acelerado.

\section{MATERIALES Y MÉTODOS}

\section{Materiales}

Plátano verde (UTROPICA, Ecuador) obtenido del mercado La Vega Central de la ciudad de Santiago en Chile y aceite de maravilla (Chef, COPRONA, Chile) fueron las materias primas.

\section{Obtención de las muestras}

Para la obtención de las muestras se siguió el protocolo establecido por Flores, 2012 (5). Para esto, el plátano verde fue lavado y pelado, se eliminaron los extremos con el fin de obtener un diámetro homogéneo a lo largo del mismo. Se cortó en rodajas de $2 \mathrm{~cm}$ de espesor que se sumergieron en aceite de maravilla a $160{ }^{\circ} \mathrm{C}$ durante 3 minutos y se escurrieron por 2 minutos, luego se formaron y almacenaron en bolsas plásticas herméticas a $-10{ }^{\circ} \mathrm{C}$ durante 12 horas.

\section{Almacenamiento de las muestras}

Se creó un ambiente con $90 \%$ de humedad relativa, en el cual se almacenaron 6 patacones identificados: 3 para evaluación de color y brillo, y 3 para pérdida de peso. Posteriormente estos se colocaron en estufas de laboratorio (VELP, Italia) a las temperaturas de experimentación $\left(5^{\circ} \mathrm{C}, 20^{\circ} \mathrm{C}\right.$ y $\left.30{ }^{\circ} \mathrm{C}\right)$.

\section{Evaluación de brillo y color}

Mediante un sistema de visión computacional que consta de una caja oscura con 4 iluminantes (D65) de $18 \mathrm{~W}$ 
(Phillips) colocados a $45^{\circ}$ de la muestra, además de una cámara digital (Canon 4 MP Powershot G3, Japón) a 22,5 $\mathrm{cm}$ de distancia de la misma (7) se tomaron imágenes digitales de ambos lados de cada uno de los patacones. Las fotografías fueron en fondo blanco y se analizaron con el programa Adobe Photoshop CS5 extended, obteniéndose los valores RGB (red: rojo, green: verde y blue: azul) que luego se convirtieron a los parámetros del sistema CIE L*a*b*. Finalmente se calculó el cambio de color $\Delta$ E2000 descrito por Sharma et. ál., 2005 (8).

\section{Porcentaje de pérdida de peso}

Las muestras fueron pesadas en diferentes momentos en una balanza analítica electrónica (Shimadzu, AUX 120, Japón) durante su almacenamiento. Los porcentajes de

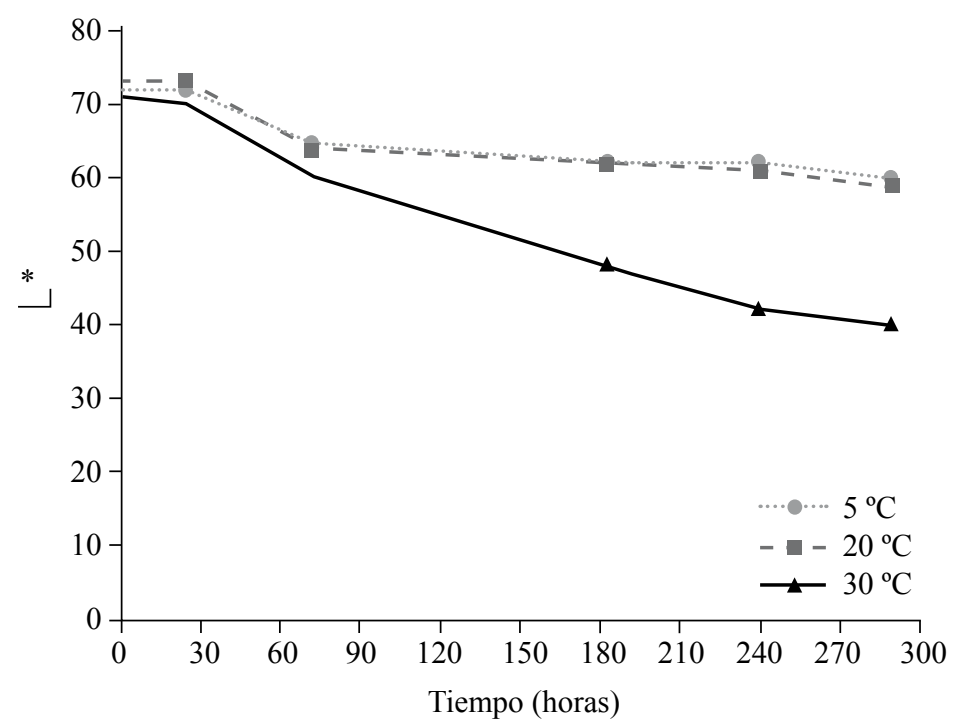

Figura 1. Cambio en el brillo de patacones prefritos congelados a distintas temperaturas de almacenamiento pérdida de peso se calcularon mediante la siguiente ecuación:

$\%$ pérdida de peso $=[(\mathrm{M} 0-\mathrm{Mt}) / \mathrm{M} 0]^{*} 100$

Donde M0 es el peso inicial de la muestra (g) y Mt el peso de la muestra a un tiempo t de tratamiento $(\mathrm{g})$.

\section{Modelamiento cinético}

Se determinó el orden de reacción y las constantes de velocidad del brillo ( $\left.\mathrm{L}^{*}\right)$, cambio del color $(\Delta \mathrm{E})$ y porcentaje de pérdida de peso a partir de los datos experimentales realizando un análisis de regresión simple para los modelos cinéticos de orden cero y orden uno de acuerdo a las siguientes ecuaciones:

$$
\begin{gathered}
\mathrm{Q}=\mathrm{Q}_{0} \pm \mathrm{kt} \text {; para orden cero } \\
\mathrm{y} \\
\mathrm{Q}=\mathrm{Q}_{0} \quad \mathrm{e}^{ \pm \mathrm{kt}} \text {; para orden uno }
\end{gathered}
$$

Donde Q es el parámetro que se degrada a un determinado tiempo $t$, Q0 el parámetro al tiempo inicial 0 , $\mathrm{k}$ es la constante cinética de la velocidad de degradación a una determinada temperatura y $\mathrm{t}$ es el tiempo. También se determinó la dependencia térmica de los mismos parámetros por medio

\begin{tabular}{|c|c|c|c|}
\hline \multirow{2}{*}{$\begin{array}{l}\text { Tiempo de almacenamiento } \\
\text { (horas) }\end{array}$} & \multicolumn{3}{|c|}{ Temperatura de almacenamiento } \\
\hline & $5^{\circ} \mathrm{C}$ & $20^{\circ} \mathrm{C}$ & $30^{\circ} \mathrm{C}$ \\
\hline 0 & & & \\
\hline$>72$ & & & \\
\hline
\end{tabular}
del cálculo de la energía de activación mediante las siguientes ecuaciones:

Tabla 1. Presencia de mohos en patacones prefritos congelados a distintas temperaturas de almacenamiento 


\section{RESULTADOS Y DISCUSIÓN}

$$
\begin{aligned}
& \mathrm{k}=\mathrm{k}_{0} \exp \left(-\frac{\mathrm{E}_{\mathrm{a}}}{\mathrm{RT}}\right) \\
& \operatorname{Ln} \mathrm{k}=\operatorname{Ln~}_{0}-\frac{\mathrm{E}_{\mathrm{a}}}{\mathrm{RT}}
\end{aligned}
$$

\section{Evaluación de brillo y color}

A las temperaturas de estudio es posible el crecimiento de microorganismos (9), este factor evidentemente contribuyó a la disminución en el brillo ( $\mathrm{L}^{*}$ ) debido a la presencia de mohos en las muestras almacenadas a $20^{\circ} \mathrm{C}$ y $30^{\circ} \mathrm{C}$ después de las 72 horas de almacenamiento aproximadamente (tabla 1). Matiacevich y col., 2011 reportaron resultados semejantes en arándano, atribuyendo este cambio del brillo a la presencia de Botrytis cinérea que tiene un crecimiento acelerado a altas temperaturas (7). Además, es conocido que la temperatura es un factor que contribuye al pardeamiento de los alimentos (10), por esta razón el oscurecimiento (disminución en el parámetro $\mathrm{L}^{*}$ ) fue mucho más evidente en las muestras almacenadas a $30{ }^{\circ} \mathrm{C}$ en comparación a las de $5^{\circ} \mathrm{C}$ y $20^{\circ} \mathrm{C}$ (figura 1). Por otro lado, en la Figura 2 se observa que las muestras almacenadas a $5{ }^{\circ} \mathrm{C}$ y $20^{\circ} \mathrm{C}$ presentaron similar cambio de los parámetros de cromaticidad, mientras que en los patacones almacenados a $30{ }^{\circ} \mathrm{C}$ existe un significativo aumento en $a^{*} y$ disminución en $b^{*}$. Resultados similares fueron obtenidos por Ammawath y col., 2002 en muestras almacenadas a $27{ }^{\circ} \mathrm{C}$, atribuyendo este cambio de color a la insaturación de carotenoides susceptibles a la oxidación que promueven la disminución del tono amarillento, propio del producto, durante el almacenamiento (11). Así y en consecuencia, el cambio de color $(\Delta \mathrm{E} 2000)$ mostró similar comportamiento y equilibrio a
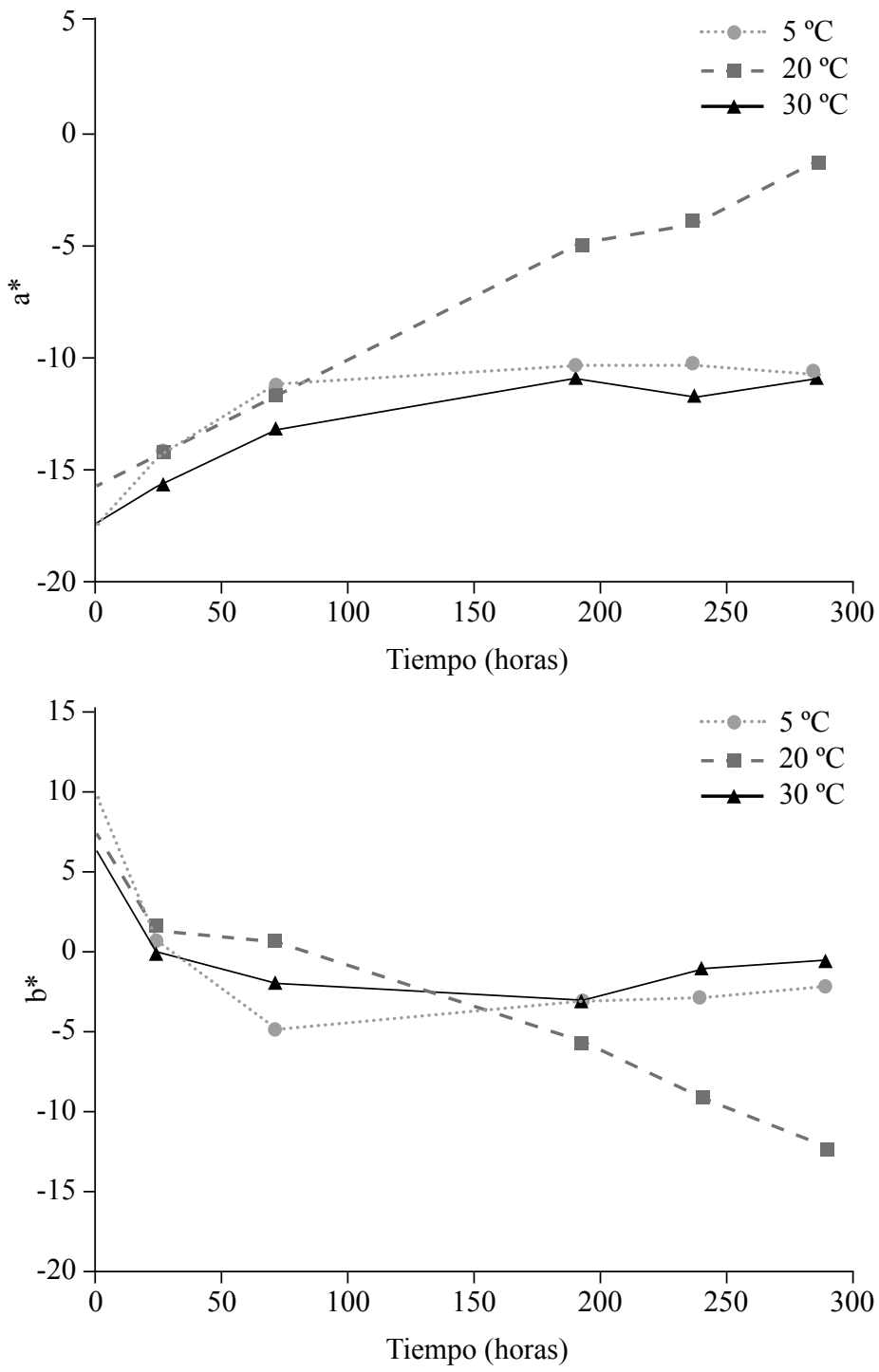

Figura 2. Cambio en los parámetros de cromaticidad de patacones prefritos congelados a distintas temperaturas de almacenamiento

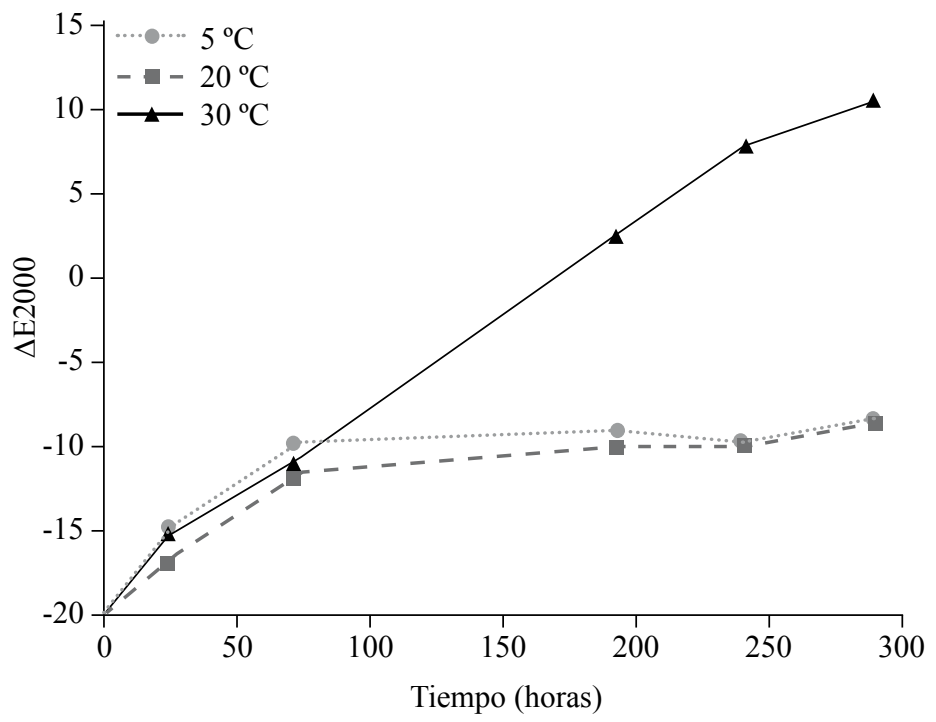

Figura 3. Modificación de color $(\Delta \mathrm{E} 2000)$ en patacones prefritos congelados a distintas temperaturas de almacenamiento 
las temperaturas de $5{ }^{\circ} \mathrm{C}$ y $20^{\circ} \mathrm{C}$ (figura 3 ). En cambio, las muestras a $30^{\circ} \mathrm{C}$ sufrieron una drástica modificación, mostrando una pendiente mucho mayor, lo cual no sería favorable en la aceptabilidad del producto.

\section{Determinación de pérdida de peso}

La Figura 4 muestra el comportamiento cinético del porcentaje de pérdida de peso en los patacones. Los valores al final de la experimentación fueron $0,51 \%, 3,01 \%$ y $7,28 \%$ para $5{ }^{\circ} \mathrm{C}, 20^{\circ} \mathrm{C}$ y $30{ }^{\circ} \mathrm{C}$, respectivamente. Es claro que el proceso de prefritura provocó únicamente una cocción superficial por lo que la actividad de agua entre $0,95-0,98$ reportada para la fruta fresca no cambió significativamente (12). Esto permitió la transferencia de humedad por transpiración (6) y la presencia de mohos (tabla 1) observada principalmente en las muestras

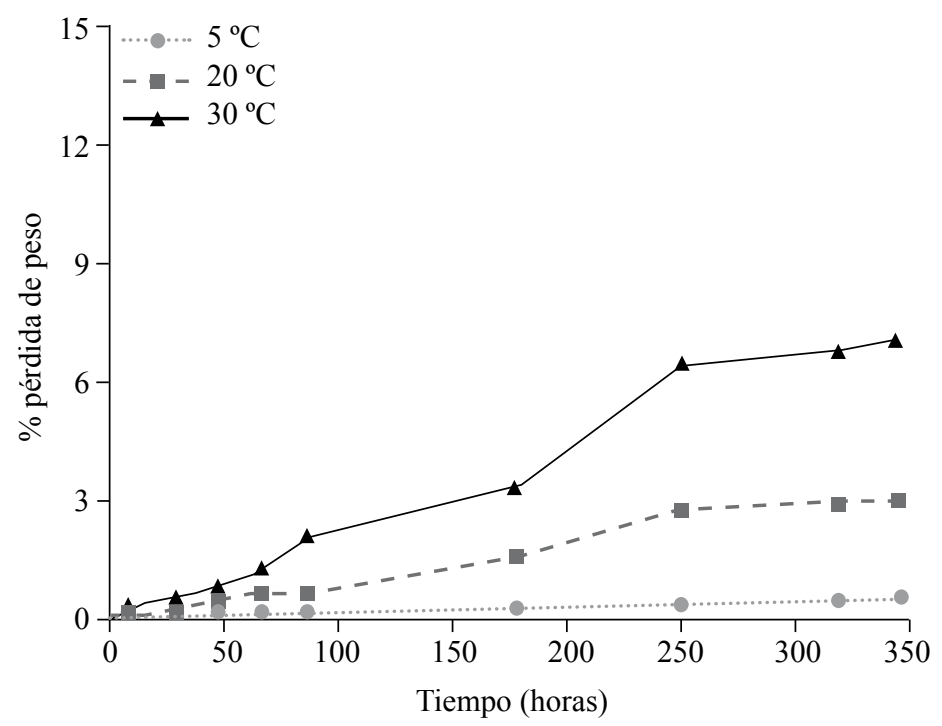

Figura 4. Porcentaje de pérdida de peso en patacones prefritos congelados a distintas temperaturas de almacenamiento almacenadas a $20{ }^{\circ} \mathrm{C}$ y $30{ }^{\circ} \mathrm{C}$. Barreiro et. al., (2006) muestran resultados de pérdida de peso para la banana entre 5,2 - 6,7\% al cabo de su periodo normal de almacenamiento en condiciones óptimas y mencionan que estos porcentajes pueden ser apreciables especialmente en tiempos largos de almacenamiento a condiciones adversas, pudiendo alcanzar cifras de hasta el $30 \%$ en casos extremos (6). En nuestro trabajo, estos valores son notablemente menores y se atribuye al procesado (prefritura) de la fruta, pues se crea una capa rígida que probablemente impida o dificulte el paso de agua presente desde el producto.

\section{Modelamiento cinético}

El resumen de los resultados se muestra en la tabla 2. De acuerdo a los coeficientes de correlación obtenidos, el brillo $\left(\mathrm{L}^{*}\right)$ siguió una cinética de primer orden, mientras que el cambio de color $(\Delta \mathrm{E})$ y porcentaje de pérdida de peso una cinética de orden cero. Las constantes de velocidad de reacción indicaron que todos los parámetros son dependientes de la temperatura de almacenamiento pues aumentan cuando más alta es esta. En el caso de L* y $\Delta$ E2000 se observaron bajas correlaciones, por lo que posiblemente se deberían aplicar modelos polinómicos con el fin de obtener un ajuste aceptable como ha sido

\begin{tabular}{|c|c|c|c|c|c|}
\hline \multirow{2}{*}{ Parámetro } & \multirow{2}{*}{ Temperatura } & \multicolumn{2}{|c|}{ Orden $\mathbf{k}$} & \multicolumn{2}{c|}{ Orden 1 } \\
\cline { 2 - 6 } & & $\mathbf{k}$ & $\mathbf{r}^{\mathbf{2}}$ & $\mathbf{k}$ & $\mathbf{r}^{\mathbf{2}}$ \\
\hline \multirow{3}{*}{$\Delta \mathrm{E} 2000$} & $5^{\circ} \mathrm{C}$ & 0,0192 & 0,6130 & 0,0192 & 0,6130 \\
\cline { 2 - 6 } & $20^{\circ} \mathrm{C}$ & 0,0278 & 0,8063 & 0,0036 & 0,7180 \\
\cline { 2 - 6 } & $30^{\circ} \mathrm{C}$ & 0,1158 & 0,9938 & 0,0072 & 0,9435 \\
\hline \multirow{3}{*}{$L^{*}$} & $5^{\circ} \mathrm{C}$ & 0,0410 & 0,8484 & 0,0006 & 0,8596 \\
\cline { 2 - 6 } & $20^{\circ} \mathrm{C}$ & 0,0466 & 0,8526 & 0,0007 & 0,8653 \\
\cline { 2 - 6 } & $30^{\circ} \mathrm{C}$ & 0,1153 & 0,9790 & 0,0021 & 0,9908 \\
\hline \multirow{3}{*}{$\begin{array}{c}\text { Pérdida de } \\
\text { peso }\end{array}$} & $5^{\circ} \mathrm{C}$ & 0,0012 & 0,9462 & 0,0077 & 0,9924 \\
\cline { 2 - 6 } & $20^{\circ} \mathrm{C}$ & 0,0091 & 0,9794 & 0,0109 & 0,9169 \\
\cline { 2 - 6 } & $30^{\circ} \mathrm{C}$ & 0,0294 & 0,9579 & 0,0107 & 0,8900 \\
\hline
\end{tabular}

Tabla 2. Orden y constantes de velocidad de reacción en patacones prefritos congelados a distintas temperaturas de almacenamiento 
el caso de varios autores quienes han ensayado modelos combinados o fraccionales con el mismo objetivo $(13,14)$.

Por otro lado, es conocido que el valor de Ea es una medida proporcional a la sensibilidad de la reacción a la temperatura (15). De acuerdo con los resultados de la tabla 3, el brillo ( $\left.\mathrm{L}^{*}\right)$ es el menos afectado por la temperatura de almacenamiento pues presenta el valor de Ea más bajo, sin embargo el cambio de color $(\Delta \mathrm{E} 2000)$ fue el más susceptible en concordancia con los resultados obtenidos en la evaluación de brillo y color.

\section{CONCLUSIONES}

Se evaluó la cinética de deterioro respecto al brillo, cambio de color y porcentaje de pérdida de peso en patacones prefritos congelados almacenados a $5{ }^{\circ} \mathrm{C}, 20{ }^{\circ} \mathrm{C}$ y $30{ }^{\circ} \mathrm{C}$. El brillo $\left(\mathrm{L}^{*}\right)$ siguió una cinética de primer orden siendo el menos dependiente de la temperatura de almacenamiento. Por el contrario, el cambio de color $(\Delta \mathrm{E} 2000)$ siguió una cinética de orden 0 indicando una gran dependencia de la

\begin{tabular}{|c|c|}
\hline Parámetro & $\mathbf{E}_{\mathbf{a}}(\mathbf{k c a l} / \mathbf{m o l})$ \\
\hline$\Delta \mathrm{E} 2000$ & 26,61 \\
\hline $\mathrm{L}^{*}$ & 7,72 \\
\hline Porcentaje de pérdida de peso & 21,45 \\
\hline
\end{tabular}

Tabla 3. Energía de activación en patacones prefritos congelados a distintas temperaturas de almacenamiento

temperatura. En ambos parámetros las muestras almacenadas a $5{ }^{\circ} \mathrm{C}$ y $20^{\circ} \mathrm{C}$ alcanzan el equilibrio después de las 72 horas. Por otro lado, existe un elevado porcentaje de pérdida de peso de los patacones a altas temperaturas y este factor sigue una cinética de orden 0 . Así, de acuerdo a los parámetros estudiados, se recomienda temperaturas hasta de $5{ }^{\circ} \mathrm{C}$ durante el almacenamiento de este producto para la conservación de sus características físicas. Sin embargo, es necesario que los parámetros involucrados en el proceso de obtención también sean estudiados.

\section{AGRADECIMIENTOS}

Los autores agradecen a la Universidad de Santiago de Chile (USACH) y al Grupo de Investigación en Propiedades de los Alimentos (INPROAL) del Departamento de Ciencia y Tecnología de los Alimentos, USACH.

1. Quiceno MC, Giraldo GA, Villamizar RH. Caracterización fisicoquímica del plátano (Musa paradisiaca sp. AAB, Simmonds) para la industrialización. Rev UGCiencia-Universidad La Gran Colomb. 2014; 20: 48-54.

2. Paz R, Pesantez Z. Potencialidad del plátano verde en la nueva matriz productiva del Ecuador. Rev Científica Yachana. 2013; 2.

3. Granados C, Acevedo D, Cabeza A, Lozano A. Análisis de perfil de textura en plátanos pelipita, hartón y topocho. Inf tecnológica. 2014; 25: 35-40.

4. Dávila K. Harina y productos de plátano. Colombia: Universidad del Valle; 2007. 34 p.

5. Flores W. Manual Técnico para el procesamiento tradicional del plátano. Costa Rica: Proyecto Fontagro FTG-7010/2007; 2012. 45 p.

6. Barreiro J, Sandoval A. Operaciones de conservación de alimentos por bajas temperaturas. Venezuela: Equinoccio; 2006.

7. Matiacevich S, Silva P, Enrione J, Osorio F. Quality Assessment of Blueberries by Computer Vision. Procedia Food Sci. 2011; 1: 421-425.

8. Sharma G, Wu W, Dalal EN. The CIEDE2000 Color-Difference Formula: Implementation Notes, Supplementary Test Data, and Mathematical Observations. Color Res Appl. 2005 Feb; 30: 21-30. 
9. Bello Gutiérrez J. Ciencia bromatológica : principios generales de los alimentos. Ediciones Díaz de Santos; 2000.

10. Pedreschi F, Bustos O, Mery D, Moyano P, Kaack K, Granby K. Color kinetics and acrylamide formation in NaCl Soaked Potato Chips. J Food Eng. 2007 Abr; 79: 989-997.

11. Ammawath W, Che Man YB, Yusof S, Rahman RA. Effects of Type of Packaging Material on Physicochemical and Sensory Characteristics of Deep-Fat-Fried Banana Chips. J Sci Food Agric. 2002; 82: 1621-1627.

12. García C, Giraldo G, Hurtado H, Mendivil C. Cinética enzimática de la polifenol oxidasa del banano Gros michel en diferentes estados de maduración. Vitae. 2006; 13: 13-19.

13. Lau MH, Tang J, Swanson BG. Kinetics of Textural and Color Changes in Green Asparagus During Thermal Treatments. J Food Eng. 2000; 45: 231-236.

14. Cortés M, Chiralt A. Cinética de los cambios de color en manzana deshidratada por aire fortificada con vitamina E. Vitae. 2008; 15: 8-16.

15. Sánchez-Chávez W, Cortez-Arredondo J, Solano-Cornejo M, Vidaurre-Ruiz J. Cinética de degradación térmica de betacianinas, betaxantinas y vitamina $\mathrm{C}$ en una bebida a base de jugo de remolacha (Beta vulgaris L.) y miel de abeja. Sci Agropecu. 2015; 111-118. 